\title{
Radiation Effects Testing at the 88-Inch Cyclotron at LBNL*
}

\author{
Margaret A. McMahan ${ }^{*}$ and Rokotura Koga \\ *Nuclear Science Division, Lawrence Berkeley National Laboratory, Berkeley, CA 94720 USA \\ " The Aerospace Corporation, El Segundo, CA USA
}

\begin{abstract}
The effects of ionizing particles on sensitive microelectronics is an important component of the design of systems as diverse as satellites and space probes, detectors for high energy physics experiments and even internet server farms. Understanding the effects of radiation on human cells is an equally important endeavor directed towards future manned missions in space and towards cancer therapy. At the 88-Inch Cyclotron at the Berkeley Laboratory, facilities are available for radiation effects testing (RET) with heavy ions and with protons. The techniques for doing these measurements and the advantages of using a cyclotron will be discussed, and the Cyclotron facilities will be compared with other facilities worldwide. RET of the same part at several facilities of varying beam energy can provide tests of the simple models used in this field and elucidate the relative importance of atomic and nuclear effects. The results and implications of such measurements will be discussed.
\end{abstract}

\section{INTRODUCTION}

As nuclear physicists, we are all familiar with the concept of energy loss, (also called stopping power or linear energy transfer (LET)), and range for ionizing particles in matter. The particle traversing material loses energy in a near linear fashion through charge-changing collisions until nearing the end of its path, at which point the remaining energy is deposited in the characteristic Bragg peak. This is the basis of the simple $\Delta \mathrm{E}-\mathrm{E} \mathrm{Si}$ telescope in use for nuclear reaction studies for many decades and the driving principle behind the choice of protons over x-rays for many of the cancer therapy facilities built in recent years. At energies below the Coulomb barrier, the atomic physics interactions leading to the Bragg curve are the main source of perturbance to the material, be it a target for a nuclear physics experiment, a detector, human tissue or a complex computer chip. For higher energy particles, nuclear reactions begin to play a role, but in general the cross sections are much less than those for the atomic properties, and are ignored to first order.

\section{RADIATION ENVIRONMENTS}

On Earth, man and his/her toys (cells, computers, detectors) are shielded from most ionizing radiation by the atmosphere and the Earth's magnetic field. The average radiation level a person receives from all sources both ionizing and non-ionizing - at sea level is $180 \mathrm{mR} /$ year. Even at ground level though, there is concern about the effects of ionizing radiation on matter, whether it be cancer clusters in areas of natural radon concentration, bit flips in memory chips of computers caused by Th contamination in the packaging [1], or potential failures of internet server farms due to neutrons reaching ground level after solar disturbances [2]. Alpha emission rates in the packaging of early Dynamic Random Access Memory (DRAM) chips often approached 60 alphas $/ \mathrm{cm}^{2}$-hr; modern DRAM processing has reduced on-chip emissions to less than $1 \times 10^{-4}$ alphas $/ \mathrm{cm}^{2}-\mathrm{hr}$. [3] At the present time, the largest contributor to single event effects in ground based electronics is from non-ionizing thermal neutrons. In interactions with boron, a common semiconductor dopant and a component of some dielectric layers, the products of the ${ }^{10} \mathrm{~B}(\mathrm{n}, \alpha)^{7} \mathrm{Li}$ reaction can cause significant radiation effects. [4]

\footnotetext{
* This work was supported by the Director, Office of Energy Research, Division of Nuclear Physics of the Office of High Energy and Nuclear Physics, of the U.S. Department of Energy under Contract No. DE-AC03-76SF00098.
} 
At the altitudes routinely flown by the larger commercial aircraft, ionizing radiation, particularly neutrons, becomes a more serious problem, both for personnel safety and the integrity of the microelectronics used in the plane. The neutrons are produced in the upper atmosphere by reactions of cosmic rays with nitrogen and oxygen. The neutron flux peaks at about $60,000 \mathrm{ft}$ with a normal flux of $1-4$ neutrons $/ \mathrm{cm}^{2} / \mathrm{sec}$, dependent on geomagnetic latitude, and is about $1 / 3$ of that value at commercial aircraft altitudes of 30-40,000 ft. [5] During a major solar disturbance these fluxes can be orders of magnitude greater and the energy spectrum shifts to lower energies. As we are nearing the peak of the latest period of solar activity, it must be remembered that modern microelectronics is much more vulnerable to damage from neutrons now than they were during the last peak eleven years ago.

Radiation-induced spacecraft anomalies have been know since the Explorer I launch on January 31, 1958, when a Geiger counter put aboard by J.A. Van Allen suddenly stopped counting. It turned out that the counter was in fact saturated by an extremely high count rate. This led to the discovery of the Van Allen belts. [6] The inner belt, at 1-3.3 Earth radii (1,000-11,000 km), contains primarily protons with energies exceeding $10 \mathrm{MeV}$. The offset between the Earth's geographical and magnetic axes causes an asymmetry in the radiation belt above the Atlantic Ocean off the Brazilian coast, allowing the inner belt to dip to an altitude of $250 \mathrm{~km}$. This "South Atlantic Anomaly" is important because it occupies a region through which low-orbiting satellites spend as much as $30 \%$ of their time. The source of protons in the inner belt is from the decay of cosmic rays, and is fairly stable, though subject to occasional perturbations due to geomagnetic storms, and large variations with the 11year solar cycle. There are more protons of all energies at solar minimum (by about a factor of 5) than at solar maximum because the cosmic rays penetrate more easily when the sun is less active. By contrast, during a solar flare, the number of protons increases by 5-6 orders of magnitude. [7]

The first spacecrafts lost due to total radiation dose effects occurred unexpectedly in 1962. A Bell Labs satellite, Telstar, was launched just one day after a high altitude nuclear test weapons test. This weapons test produced a large number of beta particles which caused a new and very intense radiation belt lasting until the early 1970's. Telstar and six other satellites were lost within a seven month period after this weapons test. Telstar was well-studied and the loss was traced directly to break down of diodes in the command decoder due to the total radiation dose. [8]

The "hardness" of an object to radiation is a major consideration in the design of satellites and space missions, both manned and un-manned. Because the loss of a piece of equipment in space can be very costly, scientists and engineers from the aerospace industry, NASA and the Department of Defense perform radiation effects studies first using sources $(\gamma, \alpha)$ and accelerators (e, n, p, heavy ions). With the proliferation of commercial satellites and the trend towards faster and cheaper design of space missions by NASA, commercialoff-the-shelf (COTS) parts are now used in place of parts designed to be radiation hard. This requires many more parts to be tested. The results of these studies must then be extrapolated to the conditions expected at the orbit and for the lifetime of the mission being designed.

\section{RET FACILITIES}

\section{Overview}

Accelerators designed for nuclear physics are ideal for radiation effects studies as well, often allowing one to test a part in a few hours to fluences well above what it would be expected to encounter in space during the lifetime of a mission. While earthbound accelerators don't span the full energy range of the particles found in space, they cover easily the range of the lower energy, more abundant particles which cause the most damage. Thus high energy accelerators are only necessary to obtain enough range to penetrate a microchip or detector to the depths at which it is sensitive to radiation.

To first order, the atomic interactions leading to radiation effects are independent of energy and dependent on the LET of the particle in the material. Scientists and engineers study either single event effects (SEE), in which the damage is caused by a single ion traversing the material, or bulk effects such as total dose. SEEs occur due to the free electron-hole pairs created by the ion's passing, which can deposit charge in unexpected and unwanted places, often leading to voltage transients on the nodes of the circuit and current transients across device junctions. SEEs include errors which are "soft" (correctable by outside control) such as upsets (SEU) (bit flips in memory chips). Other "hard" errors are destructive, causing permanent damage to the circuit. Examples include burnout (SEB) and gate rupture (SEGR) in power transistors. Other errors, for example, latch-up (SEL), can lead to either of the above conditions, depending on the severity of the circuit response. 


\section{Uniform lon Beam}

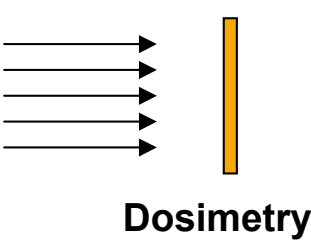

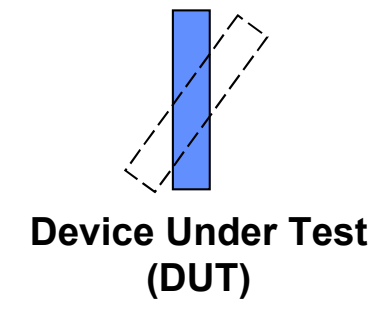

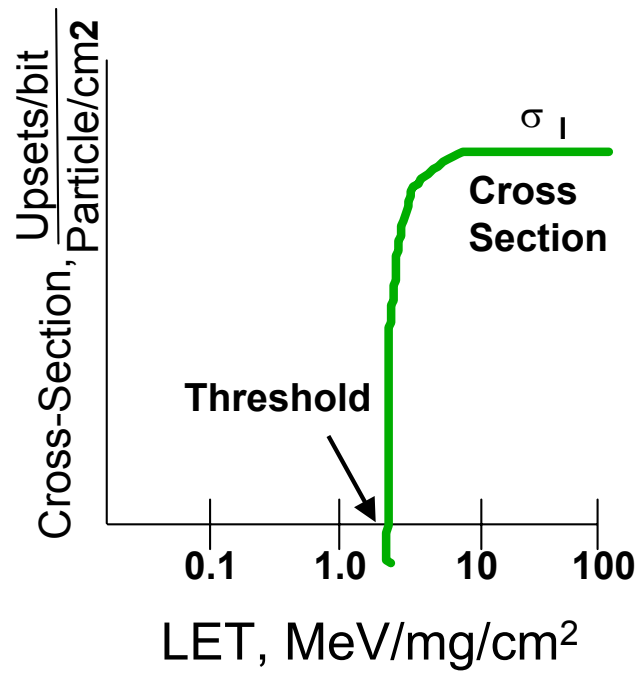

FIGURE 1. Schematic of RET set up and results

In SEE measurements, the LET is varied by changing the ion species and/or the path length through the material (achieved by rotating the sample). The cross section for the phenomena being measured has a typical behavior as shown schematically in Figure 1. There is a threshold below which no errors occur, then a steep rise with LET followed by a leveling off, or saturation, of the cross section. Beam requirements include a uniform, sometimes large, beam spot, and well-characterized dosimetry.

\section{The 88-Inch Cyclotron Facility}

The central component of the 88-Inch facility is a sector-focussed, variable-energy cyclotron fed by two Electron Cyclotron Resonance (ECR) high charge-state ion sources. Light ions $-\mathrm{p}, \mathrm{d},{ }^{3} \mathrm{He}$ and ${ }^{4} \mathrm{He}-$ are produced up to total energies of 55, 65, 135 and $130 \mathrm{MeV}$, respectively. Light heavy ions can be produced at energies up to $32 \mathrm{MeV} /$ nucleon. As the mass increases, the maximum energy per nucleon decreases. A particle nanoamp of ${ }^{209} \mathrm{Bi}$ is available up to an energy of $4.5 \mathrm{MeV} /$ nucleon. The combination of cyclotron and ECR source provides the unique ability to run "cocktails" of ions. [9] A cocktail is a mixture of ions of near-identical charge-to-mass $(\mathrm{q} / \mathrm{m})$ ratio. The ions are tuned out of the source together and the cyclotron acts as a mass analyzer to separate them, allowing one to switch from one ion to another with small adjustments of the accelerator RF. This means that the ion and therefore the linear energy transfer (LET) delivered to the component can be changed in approximately one minute. Intensity variations between the components of the cocktail are compensated for with a series of attenuator grids at the ion source which allow adjustments over nine orders of magnitude.

The four cocktail combinations most commonly in use at the 88-Inch Cyclotron are summarized in Table 1. Figure 2 plots the LET versus range for each element of the four cocktails. Certain elements of each cocktail are standard, and others can be added as needed. For example, the $4.5 \mathrm{MeV} /$ nucleon heavy ion cocktail gives a range of LET from 2.9 to $61.8 \mathrm{MeV} / \mathrm{mg} / \mathrm{cm}^{2}$ with its standard components. If a lower LET is needed, boron can be added at $1.5 \mathrm{MeV} / \mathrm{mg} / \mathrm{cm}^{2}$. If a higher LET is needed, bismuth (LET $=98.3 \mathrm{MeV} / \mathrm{mg} / \mathrm{cm}^{2}$ ) is run. The bismuth beam comes from the upgraded Advanced-ECR source (AECR-U) so the switchover takes about 30 minutes. Cocktails at intermediate energies are available.

The versatility of the ECR/cyclotron combination allows other tricks, such as quickly changing the beam energy by changing the charge state of the ions. [10] This results in expanded versions of the heavy ion cocktails which are not included in Table 1. For example, the standard $10 \mathrm{MeV} /$ nucleon cocktail contains seven elements. With expanded tuning, N, O, Si and S are available, as well as a wider range of energies for the standard components. For example, by varying the charge state of the ${ }^{136} \mathrm{Xe}$ from +34 to +42 , the energy changes from 1014 to $1544 \mathrm{MeV}$ and the LET from $52.0-57.4 \mathrm{MeV} / \mathrm{mg} / \mathrm{cm}^{2}$ over $\approx 0.7 \mathrm{MeV} / \mathrm{mg} / \mathrm{cm}^{2}$ steps. 
TABLE 1. Summary of 88 " Cyclotron cocktail beams

\begin{tabular}{|c|c|c|c|c|}
\hline Cocktail & Standard Ions & Other Ions $^{\mathrm{a}}$ & LET $\left(\mathrm{MeV} / \mathrm{mg} / \mathrm{cm}^{2}\right)$ & Range in $\mathrm{Si}$ \\
\hline $\begin{array}{l}4.5 \\
\mathrm{MeV} / \text { nucleon } \\
(\mathrm{HI})\end{array}$ & $\begin{array}{l}{ }^{15} \mathrm{~N}, \quad{ }^{20} \mathrm{Ne},{ }^{40} \mathrm{Ar}, \\
{ }^{65} \mathrm{Cu},{ }^{86} \mathrm{Kr},{ }^{136} \mathrm{Xe}\end{array}$ & $\begin{array}{l}\mathrm{HeH}^{\mathrm{b}},{ }^{10} \mathrm{~B},{ }^{59} \mathrm{Co}, \\
{ }^{78} \mathrm{Kr},{ }^{209} \mathrm{Bi}\end{array}$ & $\begin{array}{l}\text { 2.9-61.8 (standard) } \\
0.26-98.3 \text { (all) }\end{array}$ & $\begin{array}{l}43-69 \mu \text { (standard) } \\
40-180 \mu \text { (all) }\end{array}$ \\
\hline $\begin{array}{l}10 \\
\mathrm{MeV} / \text { nucleon } \\
(\mathrm{HI})\end{array}$ & 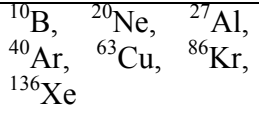 & None & $0.84-52.7$ & $115-330 \mu$ \\
\hline $\begin{array}{l}19 \\
\mathrm{MeV} / \text { nucleon } \\
(\mathrm{LI})\end{array}$ & $\begin{array}{l}\mathrm{H}_{2}{ }^{\mathrm{b}},{ }^{4} \mathrm{He},{ }^{14} \mathrm{~N},{ }^{16} \mathrm{O}, \\
{ }^{20} \mathrm{Ne},{ }^{36} \mathrm{Ar}\end{array}$ & ${ }^{2} \mathrm{H},{ }^{28} \mathrm{Si},{ }^{32} \mathrm{~S},{ }^{40} \mathrm{Ca}$ & $\begin{array}{l}0.022-6.58 \text { (standard) } \\
0.022-8.01 \text { (all) }\end{array}$ & $\begin{array}{l}0.29-2.15 \mathrm{~mm} \text { (standard) } \\
0.27-4.29 \text { (all) }\end{array}$ \\
\hline $\begin{array}{l}32.5 \\
\mathrm{MeV} / \text { nucleon } \\
\text { (LI) }\end{array}$ & $\begin{array}{l}\mathrm{H}_{2}{ }^{\mathrm{b}},{ }^{4} \mathrm{He},{ }^{14} \mathrm{~N},{ }^{16} \mathrm{O}, \\
{ }^{20} \mathrm{Ne},{ }^{36} \mathrm{Ar}\end{array}$ & ${ }^{2} \mathrm{H},{ }^{28} \mathrm{Si},{ }^{32} \mathrm{~S},{ }^{40} \mathrm{Ca}$ & $\begin{array}{l}0.014-4.46 \text { (standard) } \\
0.014-5.46 \text { (all) }\end{array}$ & $\begin{array}{l}0.69-5.56 \text { (standard) } \\
0.63-11.1 \text { (all) }\end{array}$ \\
\hline
\end{tabular}

${ }^{\mathrm{a}}$ These ions require special arrangements and advance notice.

${ }^{\mathrm{b}}$ LETs and Ranges for molecular ions are calculated for separate components after breakup in target or scattering foil.

Protons are available to a maximum energy of $55 \mathrm{MeV}$ and currents from a few hundred ions/sec up to $20 \mu \mathrm{A}$. Depending on the size of the area to be irradiated and the dosimetry method employed, total doses of $10^{15}$ ions $/ \mathrm{cm}^{2}$ can easily be obtained and higher doses are feasible. Protons are available at energies as low as 1 $\mathrm{MeV}$; special dosimetry is required below $\approx 6 \mathrm{MeV}$. The proton beams at the Cyclotron have been used for a wide variety of studies, for instance, SEE studies, radiation damage studies of CCD chips for balloon launches, damage studies of electronics and detector material for the Silicon Vertex Tracker for the ATLAS detector at the LHC, reaction studies for Boron Neutron Capture Therapy (BNCT), radiation biology studies and the study of energy loss through space suit material.

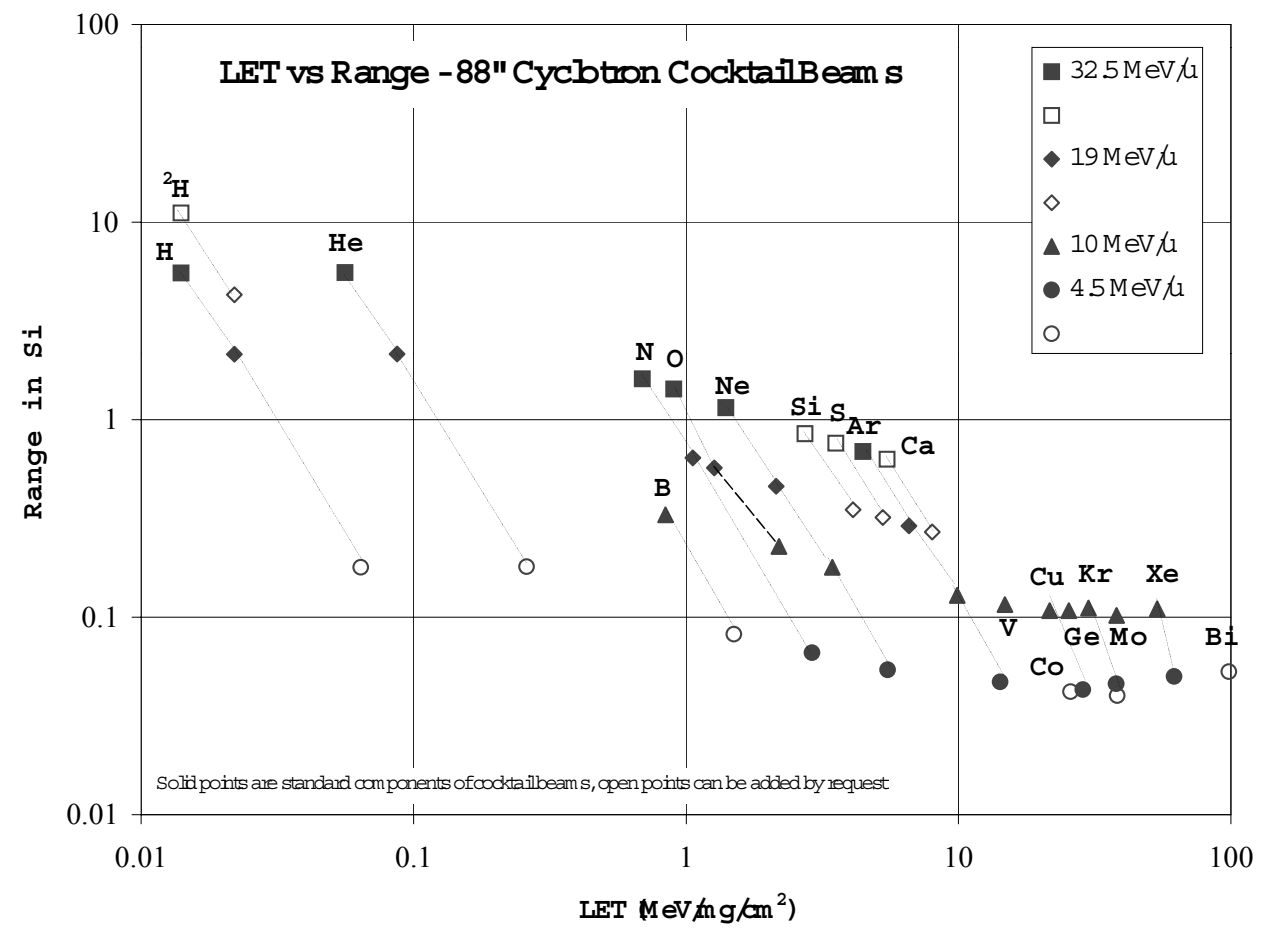

FIGURE 2. LET versus Range for 88 " Cyclotron heavy ion cocktails 


\section{Other Facilities}

The diverse needs and backgrounds of the RET community means that they rely on many different facilities to perform their testing, many of them accelerators which are primarily operated for nuclear physics. For heavy ions testing, U.S. facilites utilized are (in the order of increasing energy), the BNL Tandem, the 88" Cyclotron, the Texas A\&M Cyclotron, NSCL at MSU, and the AGS at BNL. The total beam time devoted to these studies among all the facilites is around 2000 hours/year, and brings in a small amount of supplemental income to the operating budget. Other facilities world wide include the Orsay Tandem, the Louvain-le-Neuve Cyclotron, GANIL and GSI in Europe and JAERI in Japan. Facilities available for proton irradiation in North America include the 88" Cyclotron, the U.C. Davis Cyclotron, IUCF and TRIUMF, plus a variety of proton therapy facilites with small amounts of research time. In the U.S., the main facility utilized for neutron studies is LANSCE at Los Alamos.

Traditionally, most heavy ion testing has been done at lower energies $(<10 \mathrm{MeV} / \mathrm{u})$. This has several advantages, including the availability and lower cost of facilities, negligible radioactive activation of parts, and simple dosimetry. It has disadvantages because one is required to run in vacuum and the high LET particles have limited range in the silicon. This requires "delidding" the device to be tested, that is removing the protective layer of plastic on top of the silicon. In recent years it has become more important to have higher energies available because some of the modern microchips are not easily delidded. Thus one needs enough range to get through that layer into the active layer of the chip. Recent studies suggest that additional effects could be important when going to higher energies, such as ion track structure, and geometry effects in chips with multiple layer structure.

Koga, et al, [11] in an experiment spanning years, took an identical model of a memory chip to several facilities for testing at conditions shown in Table 2, covering four orders of magnitude in energy and range. Their conclusions were the following:

- LET seems to be a useful parameter over a wide range of energies,

- Fragmentation and interaction of secondaries at high energies may be a secondary effect,

- Nuclear interactions may become a factor at very low LET (in the threshold region), and

- Similar ion interaction studies need to be carried out with more complex structures.

TABLE 2. Ions utilized for SEU testing of a SRAM

\begin{tabular}{|l|l|l|l|l|}
\hline Facility & Ions & E/A (MeV/u) & $\begin{array}{l}\text { LET } \\
\left(\mathrm{MeV} / \mathrm{mg} / \mathrm{cm}^{2}\right)\end{array}$ & Range $(\mu)$ \\
\hline 88" Cyclotron, Berkeley, CA & $\mathrm{O}, \mathrm{Ar}, \mathrm{Xe}, \mathrm{Bi}$ & $4.5-27$ & $0.9-95$ & $46-1100$ \\
\hline TASCC*, Chalk River, CAN & $\mathrm{Ni}, \mathrm{I}, \mathrm{Au}$ & $8.7-34.5$ & $9.9-91$ & $80-560$ \\
\hline NSCL, East Lansing, MI & $\mathrm{C}, \mathrm{Ar}, \mathrm{Kr}$ & $53-60$ & $0.3-10$ & $1180-5510$ \\
\hline GANIL, Caen, FR & $\mathrm{Xe}$ & $16-30$ & $34-47$ & $160-380$ \\
\hline Bevalac*, Berkeley, CA & $\mathrm{Si}, \mathrm{Fe}$ & $282-410$ & $0.6-1.7$ & $4500-38000$ \\
\hline AGS, Upton, NY & $\mathrm{Au}$ & 11,400 & 12.5 & 400.000 \\
\hline
\end{tabular}

* now closed

\section{REFERENCES}

1. T.C. May and M.H. Woods, Int. Reliability Phys Symp Proc., 33 (1978).

2. T.J. O'Gorman, IEEE TransElect. Dev 41, 553 (1994).

3. Lloyd W. Massengill, IEEE Trans Nucl Sci 43, 576 (1996).

4. R. Baumann, et al., Proc. of the IEEE Int Reliability Physics Symposium, Las Vegas, NV, 297 (1995).

5. Eugene Normand, IEEE Trans Nucl Sci 43, 461 (1996).

6. J.A. Van Allen and L.A. Frank, Nature $\underline{\mathbf{1 8 3}}, 430$ (1959).

7. J.H. Adams Jr., et al., NRL Report 4506, (1981)

8. J.S. Mayo, et al., NASA SP-32, Vol. 2, (1963).

9. M.A. McMahan, et. al., Nucl. Instr. Meth. A253, 1 (1986).

10. D.J Clark and G.J. Wozniak, Nucl. Inst. Meth., $\underline{\mathbf{A 2 9 5}}, 34$ (1990).

11. R. Koga, et al, Proc. of the IEEE Nucl \& Space Radiation Effects Conf., Williamsburg, VA (1998). 